\title{
Multiple starbursts in Blue Compact Galaxies
}

\author{
S. Recchi ${ }^{1}$, F. Matteucci ${ }^{1, \star}$, A. D’Ercole ${ }^{2, \star \star}$, and M. Tosi ${ }^{2, \star \star \star}$ \\ 1 Dipartimento di Astronomia, Università di Trieste, Via G. B. Tiepolo 11, 34131 Trieste, Italy \\ 2 Osservatorio Astronomico di Bologna, Via Ranzani 1, 40127 Bologna, Italy
}

\author{
Received 28 August 2001 / Accepted 18 December 2002
}

\begin{abstract}
In this paper we present some results concerning the effects of two instantaneous starbursts, separated by a quiescent period, on the dynamical and chemical evolution of Blue Compact Dwarf galaxies. In particular, we compare the model results to the galaxy IZw18, which is a very metal-poor, gas-rich dwarf galaxy, possibly experiencing its first or second burst of star formation. We follow the evolution of a first weak burst of star formation followed by a second more intense one occurring after several hundred million years. We find that a galactic wind develops only during the second burst and that metals produced in the burst are preferentially lost, relative to the hydrogen gas. We predict the evolution of several chemical abundances $(\mathrm{H}, \mathrm{He}, \mathrm{C}, \mathrm{N}, \mathrm{O}$, $\alpha$-elements, Fe) in the gas inside and outside the galaxy, by taking into account, in detail, the chemical and energetical contributions from type II and Ia supernovae. We find that the abundances predicted for the star forming region are in good agreement with the $\mathrm{H}$ II region abundances derived for $\mathrm{IZw} 18$. We also predict the abundances of $\mathrm{C}, \mathrm{N}$ and $\mathrm{O}$ expected for the $\mathrm{H}$ i gas to be compared with future FUSE abundance determinations. We conclude that IZw18 must have experienced two bursts of star formation, one occurred $~ 300$ Myr ago and a recent one with an age between 4-7 Myr. However, by taking into account also other independent estimates, such as the color-magnitude diagram and the spectral energy distribution of stars in IZw18, and the fact that real starbursts are not instantaneous, we suggest that it is more likely that the burst age is between 4 and 15 Myr.
\end{abstract}

Key words. galaxies: evolution - hydrodynamics - ISM: abundances - ISM: bubbles

\section{Introduction}

Blue Compact Dwarf galaxies (BCD) are characterized by compact appearance, high gas content, very blue colors and low chemical abundances. These properties are typical of unevolved systems, thus suggesting that BCDs should have suffered very few bursts of star formation during their lives and that some of them are probably experiencing their first burst (Searle \& Sargent 1972). In a recent review, Kunth \& Östlin (2000) argued that, despite a few remaining young galaxy candidates (like IZw18, SBS 0335052 or HS $0822+3542$; Lipovetsky et al. 1999; Kniazev et al. 2000), in most BCDs an old underlying stellar population does exist, revealing at least another burst of star formation (SF) besides the present one. In a recent survey of BCDs, Östlin et al. (2001) have found that, although the young burst population dominates the integrated

\footnotetext{
Send offprint requests to: S. Recchi, e-mail: recchi@ts . astro.it

* e-mail: matteucci@ts.astro.it

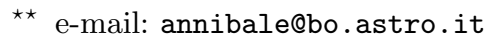

$\star \star \star$ e-mail: tosi@bo.astro.it
}

optical luminosities, it contributes only marginally to the total stellar mass. It is possible that each starburst episode in BCDs is followed by a quiescent (or almost quiescent) period, with a time scale of the order $10^{8}$ to $10^{9} \mathrm{yr}$ (Leitherer 2001), during which winds produced by supernova explosions expel the gas out of the region and the star formation fades. When the luminosity of the burst is no longer able to sustain the wind, the gas should cool and collapse back toward the center of the galaxy, thus allowing for the onset of a new burst (Babul \& Rees 1992; D'Ercole \& Brighenti 1999). Off-centered SN explosions can also drive inward-propagating shocks, thus creating the conditions of a new SF event in the center of the galaxy (Mori et al. 2001).

The galaxy IZw18 is the most metal-poor local galaxy known so far and was considered until recently as the best candidate for a truly "young" galaxy. Stellar population analyses by Hunter \& Thronson (1995) and Dufour et al. (1996) were not deep enough to reveal any old stellar population, but recent studies of deeper Color-Magnitude Diagrams (CMD), both in the optical (Aloisi et al. 1999; hereafter ATG) and in the infrared (Östlin 2000) revealed 
the presence of two stellar populations in IZw18: a young population with an age of $\sim 15 \mathrm{Myr}$ and an asymptotic giant branch population with an age of several $10^{8}$ years. Chemical evolution models by Kunth et al. (1995) fit the abundances observed in IZw18 with one, or at maximum two short bursts. Evolutionary population synthesis models by Mas-Hesse \& Kunth (1999; hereafter MHK) showed that the present burst is very young (ranging between 3 and $13 \mathrm{Myr}$, depending on whether the burst is instantaneous or continuous) and the contribution of the stars of an ancient burst to the emission over the whole UV-optical range, if any, is negligible. Legrand (2000) and Legrand et al. (2000) proposed instead a low and continuous SF regime for IZw18; furthermore they assumed that the observed metals cannot result from the material ejected by the aging starburst, because these metals are hidden in a hot phase and therefore undetectable with optical spectroscopy.

In a previous paper (Recchi et al. 2001; hereafter Paper I) we presented a study concerning the effect of a single, instantaneous starburst on the dynamical and chemical evolution of a gas-rich dwarf galaxy, with galactic parameters resembling those of IZw18. We showed that the observed abundances of IZw18 could also be reproduced by a single-burst model provided that its age is $\sim 30 \mathrm{Myr}$, occurring in a primordial gas (zero initial metallicity), although we did not exclude the presence of an underlying older population which only slightly polluted the ISM (less than 1/100 of solar metallicity). However, the estimate of $\sim 30$ Myr for IZw18 seems to be in contrast both with the spectral energy distribution and the color-magnitude diagram, as mentioned above.

Other results of Paper I can be summarized as follows:

i) A galactic wind develops as a consequence of the starburst and mostly carries away the metals produced during the starburst. In particular, we found that the metals produced by type Ia $\mathrm{SNe}$ are lost even more efficiently than the metals produced by type II SNe. This fact is important since different SN types produce different elements; in particular, the net effect is to enhance the $\alpha$-element over Fe ratio inside the galaxy relative to the gas lost from the galaxy.

ii) The cooling of metals in the gas was found to be very efficient so that most of the metals should be found in the cold gas phase already a few Myr after the beginning of the burst. Both results i) and ii) depend on the assumed energy transferred from SNe into the interstellar medium (ISM), a crucial parameter in galaxy evolution studies.

In this paper we intend to test the hypothesis of multiple starbursts with our model. In order to investigate this hypothesis, we performed some numerical simulations to study in detail the dynamical and chemical evolution of a gas-rich dwarf galaxy experiencing two single, instantaneous starbursts. Our approach makes use of a twodimensional dynamical model coupled with detailed chemical enrichment from both type II and type Ia SNe.

In Sect. 2 we summarize the properties of IZw18; in Sect. 3 we describe the model and the assumptions adopted in our simulation. The results are presented and discussed in Sect. 4. Finally some conclusions are drawn in Sect. 5 .

\section{IZw18}

The blue compact dwarf galaxy IZw18 (also known as Mrk 116) is the most metal-poor galaxy known locally (the metallicity is $\sim 1 / 50 Z_{\odot}$ ). More metal-deficient BCDs have not been found in spite of extensive surveys (Masegosa et al. 1994; Terlevich et al. 1996). The colors of this galaxy are exceptionally blue; the most recent estimates give $U-B=-0.88$ and $B-V=-0.03$ (van Zee et al. 1998), thus suggesting the presence of a dominant very young population (van Zee 2001). Given the low degree of pollution, IZw18 is also an ideal laboratory to measure the primordial helium abundance (Olive et al. 1997; Skillman et al. 1998; Izotov et al. 1999).

The morphology of IZw18 is rather complex, consisting of a "peanut-shaped" main body, formed by two starbursting regions, associated with two $\mathrm{H}$ II regions (Dufour et al. 1996). To the north and west of the main body, there are several diffuse features noted from the deep ground-based imagery of Davidson et al. (1989) and Dufour \& Hester (1990), but only the nearest of these objects (component C in the nomenclature of Davidson et al. 1989, located $22^{\prime \prime}$ NW of the NW region) is a blue irregular galaxy physically associated with the main body, while the others are background galaxies (Dufour et al. 1996). For simplicity, we focus our attention only on the main body features (see Paper I for more references).

The dynamical mass and the $\mathrm{H}$ I mass of the main body of IZw18 are of the order of $\sim 10^{8} M_{\odot}$ and $\sim 10^{7} M_{\odot}$, respectively (see e.g. Lequeux \& Viallefond 1980; Davidson \& Kinman 1985; Viallefond et al. 1987; Petrosian et al. 1997; van Zee et al. 1998). The H I column density is as high as $N_{\mathrm{H} \text { I }} \simeq 2 \times 10^{21} \mathrm{~cm}^{-2}$ (Vidal-Madjar et al. 2000).

Abundances derived from $\mathrm{H}$ II regions are reasonably well known (Searle \& Sargent 1972; Dufour et al. 1988; Skillman \& Kennicutt 1993; Garnett et al. 1995; Garnett et al. 1997; Izotov \& Thuan 1999; Izotov et al. 1999; Izotov et al. 2001a), and indicate a low content of oxygen but a rather high $\mathrm{N} / \mathrm{O}$ ratio. However, there is still some debate in the literature about the abundances in the H I region of IZw18. The first measurement of this kind, by Kunth et al. (1994), indicated a metallicity which was a factor of 20 lower than in the H II region, although the UV absorption lines they used were saturated, so this result remains still uncertain (Pettini \& Lipman 1995). Results by Pettini \& Lipman (1995) and van Zee et al. (1998) seem to indicate indeed a metallicity comparable to the abundance in the H II regions. A recent work of Levshakov et al. (2001) about argon and silicon emission line profiles in $\mathrm{IZw}_{\mathrm{w}} 18$ also reveals a good degree of mixing between hot and cold regions. Future FUSE abundance determinations will certainly shed more light on the problem. 


\section{The model}

The dark matter content, the interstellar medium (ISM) initial mass and the mass of stars formed during the first burst are shown in Table 1, and are chosen to match the observed values of IZw18. We assume that the dark matter has a quasi-isothermal distribution with a flat profile in the center (see Paper I). In fact, Borriello \& Salucci (2001) have recently shown that rotation curves in dwarf galaxies, as well as in spiral galaxies, are consistent with a flat central dark matter distribution.

We assume that the first burst of SF produces $10^{5} M_{\odot}$ of stars, distributed according to a Salpeter IMF, with an upper mass limit of $40 M_{\odot}$ and a lower mass limit of $0.1 M_{\odot}$. We follow the dynamical and chemical evolution of this first burst of SF up to $300 \mathrm{Myr}$ (model M300) and up to $500 \mathrm{Myr}$ (model M500), solving the set of gas-dynamical equations in two dimensions described in Paper I. According to the results of ATG, who suggested that the stars of the present burst could have formed with a flat IMF, we consider also an IMF with a slope $x=0.5$ (model M300F). The model parameters are shown in Table 2 .

The nucleosynthesis prescriptions are summarized in Table 3. The initial metallicity of the gas is set to zero. The adopted set of yields is from Woosley \& Weaver (1995; hereafter WW) case B and metallicity $1 / 100 Z_{\odot}$ for massive stars, whereas for low and intermediate-mass stars (IMS) we consider both the results of Renzini \& Voli (1981; hereafter RV81), and the more recent set of yields by van den Hoek \& Groenewegen (1997; hereafter VG97), which has been successfully tested by chemical evolution models of the Galaxy (Chang et al. 1999; Romano et al. 2000). One of the main differences between the two sets of yields is the assumption about the mass loss scaling parameter $\eta_{\mathrm{AGB}}$ (Reimers 1975): in RV81 most of the models are calculated assuming $\eta_{\mathrm{AGB}}=1 / 3$, while VG97 consider higher mass loss efficiencies, resulting in lower $\mathrm{CNO}$ yields. The adopted mixing length parameter for low and intermediate-mass stars $\alpha_{\mathrm{ML}}$ is different from zero, in order to allow the production of primary nitrogen in intermediate-mass stars. Concerning type Ia SNe, we adopt the yields of Nomoto et al. (1984; hereafter NTY).

The initial abundances of the second stellar generation are simply the abundances of the cold gas in the central region (a sphere of $\sim 200 \mathrm{pc}$ of radius) at the onset of the second burst (see Table 2), which result from the pollution of the first burst. We follow the evolution, in space and time, of several chemical elements of particular astrophysical interest (namely $\mathrm{H}, \mathrm{He}, \mathrm{C}, \mathrm{N}, \mathrm{O}, \mathrm{Mg}, \mathrm{Si}, \mathrm{Fe}$ ). The supernova (Ia and II) rates and the energetic prescriptions (explosion energy, thermalization efficiencies) are those adopted in Paper I, namely $\eta_{\mathrm{II}}=0.03$ and $\eta_{\mathrm{Ia}}=1$, where with $\eta_{\text {II }}$ and $\eta_{\text {Ia }}$ we indicate the efficiency of energy transfer from SNe into the ISM. The reason for this choice is that SNeII explode in a cold and dense medium, thus radiating away most of their internal blast wave energy (cooling is
Table 1. First burst of star formation.

\begin{tabular}{cccc}
\hline$M_{*}^{\mathrm{I}}\left(M_{\odot}\right)$ & $M_{\mathrm{g}}\left(M_{\odot}\right)$ & $M_{\mathrm{h}}\left(M_{\odot}\right)$ & $Z_{\mathrm{i}}\left(Z_{\odot}\right)$ \\
\hline $10^{5}$ & $1.7 \times 10^{7}$ & $6.5 \times 10^{8}$ & 0 \\
\hline
\end{tabular}

Notes: $M_{*}^{\mathrm{I}}$ is the mass of stars formed in the first episode of $\mathrm{SF}, M_{\mathrm{g}}$ is the mass of gas in the galactic region, $M_{\mathrm{h}}$ is the total mass of the dark halo and $Z_{\mathrm{i}}$ is the initial metallicity.

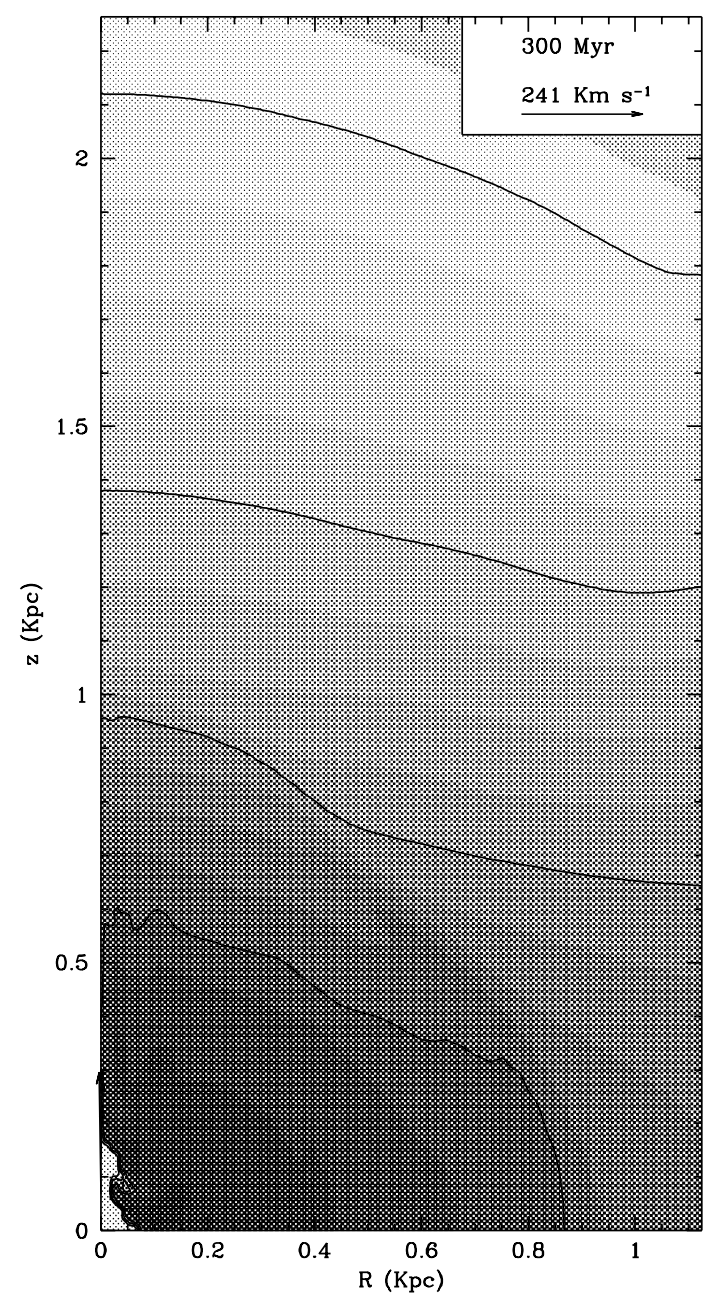

Fig. 1. Initial gas density profiles of model M300. The density scale is logarithmic and varies linearly between -29 and -23.5 .

proportional to the square of the density), whereas type Ia SNe occur after a minimum time delay of $\sim 30 \mathrm{Myr}$ in an already hot and diluted medium, thus transferring most of their energy into the ISM.

Before the onset of the second burst we checked if the physical conditions of the gas allowed new star formation to set in. In particular, we controlled whether cold gas was present in the central region and whether its column density was larger than the threshold value for SF $\left(\sim 10^{21} \mathrm{~cm}^{-2}\right)$, as suggested by Skillman et al. (1988) and Saitō et al. (1992). We ran the simulations for $\sim 150 \mathrm{Myr}$ from the beginning of the second burst for all the models M300, M300F and M500. 
Table 2. Model parameters.

\begin{tabular}{lcccccc}
\hline Model & Onset II burst (Myr) & IMF slope & $\Delta t(\mathrm{Myr})$ & $\log N_{\mathrm{H}}\left(\mathrm{cm}^{-2}\right)$ & $M_{*}^{\mathrm{II}}\left(M_{\odot}\right)$ & $Z_{\mathrm{i}}\left(Z_{\odot}\right)$ \\
\hline M300 & 300 & -2.35 & 159 & -21.5 & $5.8 \times 10^{5}$ & $1 / 50$ \\
M300F & 300 & -1.5 & 171 & -21.8 & $4 \times 10^{5}$ & $1 / 40$ \\
M500 & 500 & -2.35 & 154 & -21.4 & $6.25 \times 10^{5}$ & $1 / 100$ \\
\hline
\end{tabular}

Notes: $\Delta t$ is the duration of the second burst; $N_{\mathrm{H}}$ is the peak of column density in the central region at the onset of the second burst, $M_{*}^{\mathrm{II}}$ is the mass of stars formed in this episode of $\mathrm{SF}$ and $Z_{\mathrm{i}}$ is the mean metallicity in this region.

Table 3. Chemical prescriptions.

\begin{tabular}{cccccc}
\hline Case & SNII yields & IMS yields & SNIa yields & $\alpha_{\mathrm{ML}}$ & $\eta_{\mathrm{AGB}}$ \\
\hline $\mathrm{R}$ & $\mathrm{WW}\left(Z=1 / 100 Z_{\odot}\right)$ & RV81 & NTY & 1.5 & 0.333 \\
$\mathrm{~V}$ & $\mathrm{WW}\left(Z=1 / 100 Z_{\odot}\right)$ & VG97 & NTY & 2.0 & 4 \\
\hline
\end{tabular}

References: WW Woosley \& Weaver (1995) (models with $Z=0.01 Z_{\odot}$ ); RV81 Renzini \& Voli (1981); VG97 van den Hoek \& Groenewegen (1997); NTY Nomoto et al. (1984).

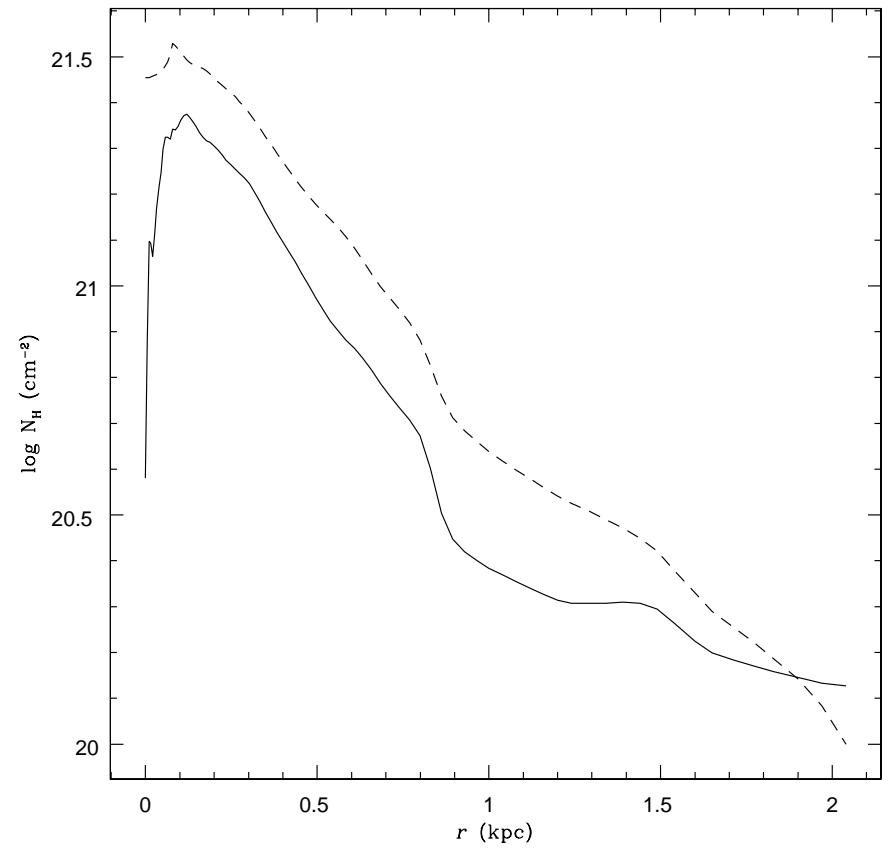

Fig. 2. Column density of the initial ISM seen edge-on (dashed line) and face-on (solid line).

\section{Results}

\subsection{Dynamical results}

\subsubsection{Model M300}

Owing to the low luminosity of the first burst $(\sim 3 \times$ $10^{37} \mathrm{erg} \mathrm{s}^{-1}$ during the SNII phase, a factor $\sim 10$ lower during the SNIa phase), a galactic wind still does not develop after 300 Myr. A cold, dense shell with dimensions $\sim 200 \times 100$ pc forms and outside this region the ISM is practically unperturbed (see Fig. 1). Inside the central region, after $300 \mathrm{Myr}, 5.8 \times 10^{6} M_{\odot}$ of cold gas $\left(<2 \times 10^{4} \mathrm{~K}\right)$ are present. The mean metallicity inside this region is $\sim 1 / 50 Z_{\odot}$, whereas in the whole galaxy (roughly an ellipsoid with dimensions $700 \times 1000 \mathrm{pc}$ ) the gas is more diluted (the mean abundance is $\sim 1 / 500 Z_{\odot}$ ). Most of the metals in the star formation region are cold (more than $99 \%$ of metals have $T<2 \times 10^{4} \mathrm{~K}$ ). This is because the superbubble evolution is slow and no shell rupture occurs. For this reason the internal cavity can cool significatively (see also Paper I).

The column density in the central region largely exceeds the threshold density (see Fig. 2). Therefore a new SF event can develop with a pre-enrichment of $1 / 50 Z_{\odot}$. Assuming an efficiency of SF of 0.1 , we obtain for this second burst $\sim 5.8 \times 10^{5} M_{\odot}$ of stars, in agreement with the mass of stars deduced for IZw18 by assuming an episode of continuous star formation lasting $13 \mathrm{Myr}$ (MHK).

We assume, in this second burst, a thermalization efficiency slightly higher than that adopted for the first burst (and in Paper I): in particular we assume $\eta_{\mathrm{II}}=0.05$. In fact, the very central regions are hot and rarefied, owing to the activity of the first generation of stars, thus a higher value of this efficiency should be expected. We should however consider that star formation is more likely to be found in the cold and dense shell, rather than in the central region where the gas is depleted. The physical conditions of the shell suggest indeed a value of the thermalization efficiency around 0.05, computed as described in Bradamante et al. (1998).

The dynamical evolution of this model is shown in Fig. 3. Because of the thermodynamical conditions of the central region and the value of the thermalization efficiency, the impact of the energetic input of the second generation of stars on the ISM dynamics is rather strong. In fact, a breakout occurs already after $30 \mathrm{Myr}$ and the 
gas produced by this second generation of stars is easily channelled along the galactic chimney.

The most relevant dynamical results of Paper I indicated a selective loss of metals, in the sense that the metals produced during the burst were ejected more easily than the gas originally present, and a fast cooling of metals, due to the slow evolution of the superbubble, was present. In this model the selective loss of metals is confirmed: at the end of the simulation $(\sim 150 \mathrm{Myr}$ after the onset of the second burst) $\sim 75 \%$ of pristine gas (not processed through stars) is lost, while the galaxy looses $\sim 87 \%$ of the metals produced in the first generation of stars and $\sim 92 \%$ of those produced in the second generation.

Differences between ejection efficiencies of SNIa and SNII products are still present: SNIa products are more easily channelled along the galactic funnel, but this effect is less evident compared to the single-burst model. In fact, the SNII products in this case are lost more easily than in the one-burst case. This is partly due to the fact that we assume a larger $\eta_{\text {II }}$ than in the one-burst case and partly by the fact that a cavity is already carved by the first burst when the second burst sets in. Both these effects favor a larger loss of metals from type II SNe. As a consequence of this, the fraction of cold metals inside the galactic region is around $77 \%$ after $\sim 20 \mathrm{Myr}$, while in the single-burst model, with a slower evolution, this fraction was around $95 \%$.

\subsubsection{Model M500}

We now follow the hydrodynamical evolution of the first burst of star formation up to 500 Myr. The evolution in this first phase is the same as in model M300. The cavity is only slightly narrower and more elongated along the polar axis; consequently the bubble slightly deflates and a part of the pristine gas outside the bubble moves toward the center, along the $R$-direction. Owing to this, the gas mass in the star forming region is a little larger than in model M300 $\left(\sim 6.25 \times 10^{6} M_{\odot}\right)$ and the metallicity is reduced $\left(Z \sim 1 / 100 Z_{\odot}\right)$. The mass of stars formed in this second burst is then $6.25 \times 10^{5} M_{\odot}$.

After the onset of the second burst, the evolution of this model is similar to that of model M300; a breakout quickly occurs (after $\sim 30 \mathrm{Myr}$ ) and the metals produced in this second burst are easily lost. Approximately $80 \%$ of metals are in a cold phase after $20 \mathrm{Myr}$, while if we consider only products of the second generation of stars, this fraction reduces to $70 \%$. The fraction of unprocessed ISM ejected in this model is $63.6 \%$, while the ejection efficiencies are $\sim 81 \%$ for the first generation of stars and $\sim 91 \%$ for the second generation.

\subsubsection{Model M300F}

In this model, owing to the flatter IMF, more SNeII are produced. At the end of the first burst less gas is present in the central region $\left(\sim 4 \times 10^{6} M_{\odot}\right)$. The stellar population is strongly biased toward massive stars and $\sim 80 \%$ of the total stellar mass is in the form of stars more massive than $2 M_{\odot}$ (more than twice of what estimated by MHK). The total number of type II SNe is around $1.3 \times 10^{4}$, which is three times the number of SNeII in model M300.

The temporal evolution of model M300F is shown in Fig. 4. At the onset of the second burst (left panel), a weak galactic wind is already present, at variance with model M300, in which most of the ISM is still unperturbed (Fig. 1). This is due to the fact that the mean luminosity of the first burst is three times higher than in model M300 ( $\sim 10^{38} \mathrm{erg} \mathrm{s}^{-1}$ during the SNII phase). The impact of the second burst of $\mathrm{SF}$ is thus even stronger than in previous cases and a breakout occurs already after $\sim 15 \mathrm{Myr}$.

The luminosity of the second burst is rather high and consequently the ejection efficiencies, computed at the end of the simulation, are close to unity. In particular, $81 \%$ of the unprocessed gas, $86.7 \%$ of metal produced by the first generation of stars and $93.9 \%$ of the metals produced by the second stellar generation are ejected out of the galactic region.

\subsection{Chemical evolution results}

The evolution of carbon, nitrogen and oxygen for model M300 (cases R and V) and for model M300F (case V only), compared to the results of the single-burst model described in Paper I, are shown in Fig. 5. We have plotted also the evolution of N/O for a model (model NP), similar to model M300 (case R), but in which we assume an "ad hoc" production of primary nitrogen from massive stars. This possibility seems to be suggested by some stellar evolution models with rotation (see for example Heger \& Langer 2000). However, precise yields calculations do not seem to exist, thus we adopted some "ad hoc" prescriptions in order to reproduce the observed N/O in IZw18. In particular, we assumed that nitrogen production in massive stars is $1 / 1000$ of the initial mass of the star, for the whole range $8 \div 40 M_{\odot}$ both in the first and in the second burst.

The abundances shown in this plot refer to the abundances of the cold $\left(T<2 \times 10^{4} \mathrm{~K}\right)$ gas inside the galactic region extending for $1 \mathrm{kpc}$ in the radial direction and for $700 \mathrm{pc}$ in the vertical direction. They should be compared with the observed $\mathrm{H}$ II region abundances. The abundances of gas in a hotter phase are, in fact, virtually undetectable (see Paper I for more details). We also computed the mean metallicity over a region larger than the galactic one and covering the whole computational grid (roughly $5 \mathrm{kpc} \times$ $2 \mathrm{kpc}$ ) and found a metallicity which can be attributed to the $\mathrm{H}$ I gas of $Z_{\mathrm{HI}} \simeq 1 / 100 Z_{\odot}$, although this estimate is rather uncertain with our model. For this even colder gas, we computed also the column densities, integrating along all the lines of sight, assuming the galaxy to be edge-on (Martin 1996). We found that the abundance ratios of the metals (showed in Fig. 6) are roughly a factor of 2 lower than those predicted for the $\mathrm{H}$ II region. These values 

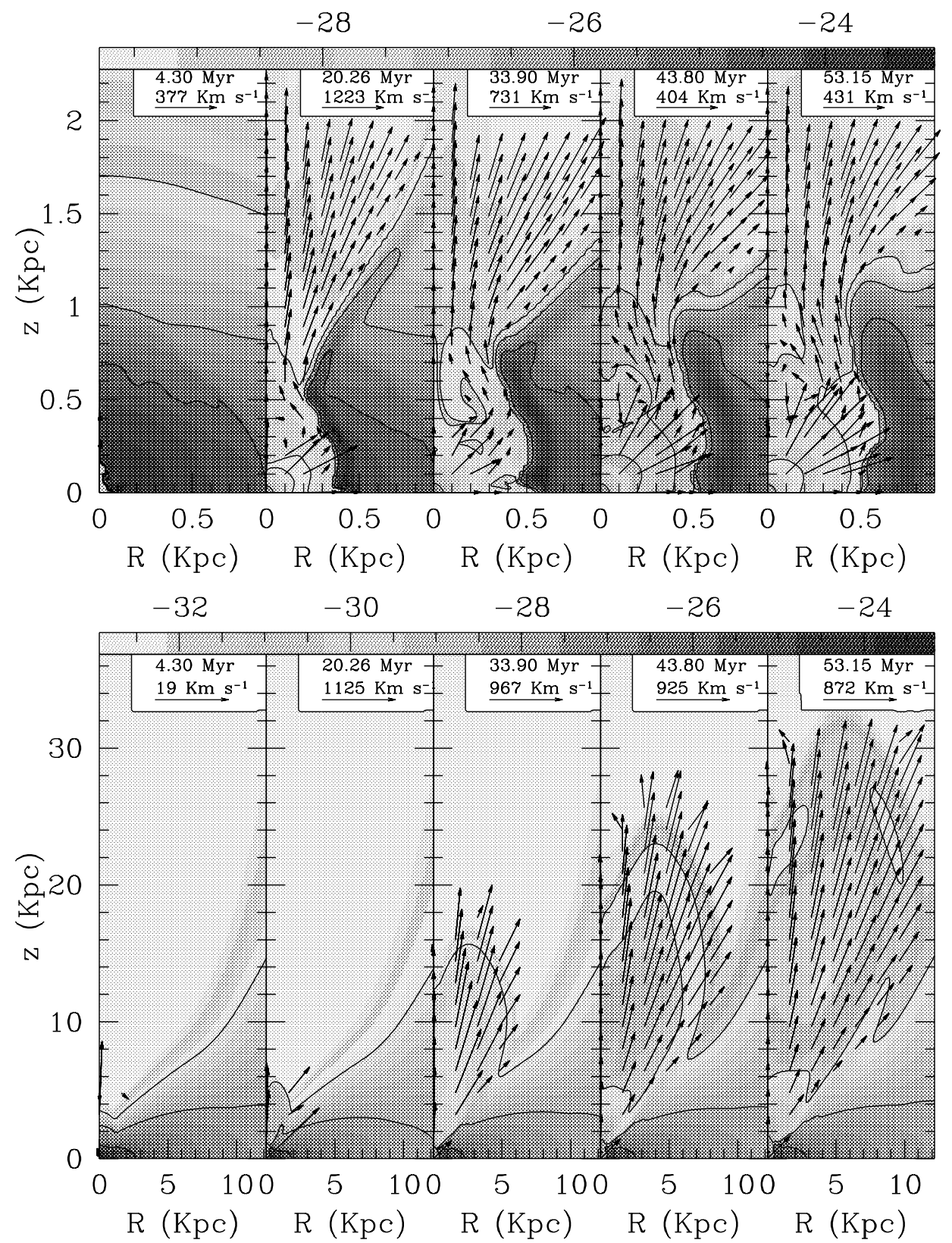

Fig. 3. Density contours and velocity fields for model M300 at different epochs (the time labelled on top of each panel refers to the time elapsed since the beginning of the second burst). The density scale (logarithmic) is given in the strip on top of the figures. The lower panels show the whole evolution of the system, whereas the upper panels are a zoom in the central regions. In order to avoid confusion, we draw only velocities with values greater than $1 / 10$ of the maximum value. This is true also for Fig. 4.

can be used for future comparison with observations (in particular they could be directly compared with FUSE/HST observations).

Coming back to Fig. 5, the superimposed dashed area represents the abundances observed in IZw18 HII regions (Dufour \& Hester 1990; Skillman \& Kennicutt 1993; Izotov et al. 1997; Garnett et al. 1997; Vilchez \& IglesiasPáramo 1998; Izotov \& Thuan 1999). We find that the single-burst model, occurring in a primordial gas at an age of $\sim 31$ Myr (case $\mathrm{R}$ ) can reasonably reproduce the abundances measured in IZw18. However, as evident in Fig. 5, the correct $\mathrm{N} / \mathrm{O}$ ratio would last only for a very short time (of the order of a couple of Myr) since for $t \gtrsim 31$ Myr the $\mathrm{N} / \mathrm{O}$ ratio will start to increase, due to the $\mathrm{N}$ produced by intermediate and low mass stars, outside the permitted observational range. Moreover, this age estimate is inconsistent with other independent estimates derived from observations (see Sect. 5).

In model M300, at the onset of the second burst, the metallicity in the central region is $\sim Z_{\odot} / 50$, and a very good agreement with the observed abundances is obtained, especially for the $\mathrm{O}$ abundance and the $\mathrm{C} / \mathrm{O}$ ratio, for a larger range of burst ages. The yields of VG97 produce substantially less $\mathrm{CNO}$, compared to what is 


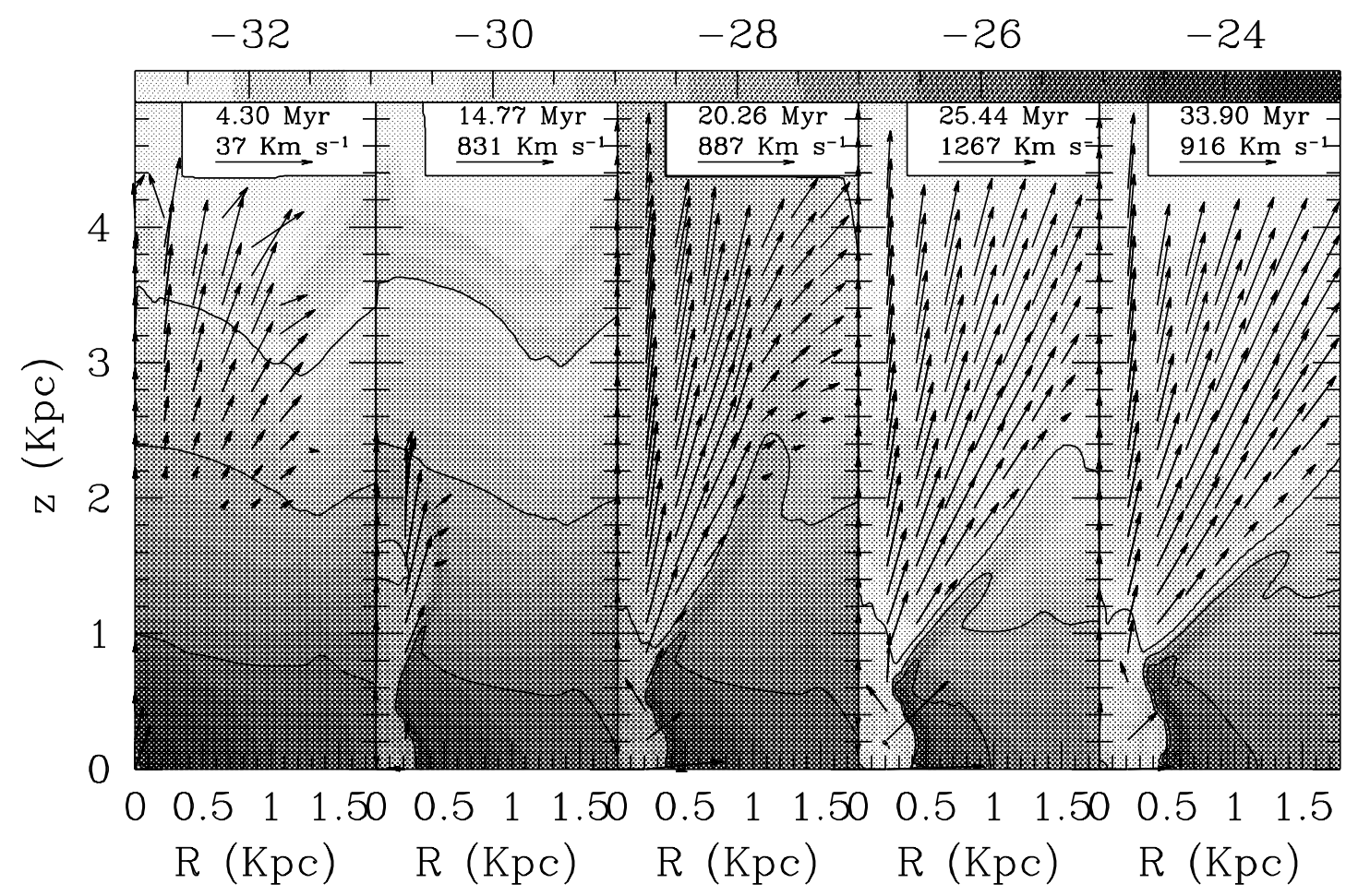

Fig. 4. Density contours and velocity fields for model M300F at different epochs.

obtained by RV81. Therefore, the evolution of nitrogen is strongly affected by the choice of nucleosynthetic prescriptions, whereas the oxygen evolution is almost identical, because $\mathrm{O}$ is mostly produced by type II SNe (see Fig. 5, upper panels).

For the model M300 we find two solutions reproducing the observed N/O abundance ratio. The first is around 5-6 Myr after the beginning of the second burst and is almost independent of the the adopted nucleosynthetic prescriptions. The second solution predicts a larger burst age and depends on the adopted set of chemical yields: in the $\mathrm{R}$ case, the N/O ratio observed in IZw18 is reproduced after a time between 20 and $40 \mathrm{Myr}$ from the onset of the second burst, whereas in case $\mathrm{V}$ we need an age between $\sim 45$ and $\sim 70 \mathrm{Myr}$ to fit the observed abundance ratios. Unfortunately, both these burst age estimates are too large and inconsistent with the spectral energy distribution. The M300F model with a flatter IMF produces much more oxygen in the first burst and indicates that the only possible solution requires an extremely short age of the second burst (around $4 \mathrm{Myr}$, in agreement with the results of MHK).

These results depend also on the thermalization efficiency, in the sense that if we assume a higher $\eta_{\mathrm{II}}$, the galactic wind is stronger and more metals are lost through the galactic chimney, thus diminishing the abundances inside the galaxy. In the limiting case $\eta_{\mathrm{II}}=1$ we have shown in Paper I that the galaxy is quickly devoided of gas, at variance with what observed in IZw18. Thus the thermalization efficiency could be higher than the assumed value, but not high enough to empty the galaxy of gas.
The thermalization efficiency of SNe, in particular type II $\mathrm{SNe}$, is a crucial parameter and a more refined model will be presented in a future paper (Recchi et al., in preparation).

It is worth noting that we assumed mainly secondary $\mathrm{N}$ production from massive stars, as implied by the adopted yields of WW, and this means a slow growth of $\mathrm{N}$ with time. However, Izotov \& Thuan (1999) and Izotov et al. (2001b) found that some low metallicity BCDs show a constant $\mathrm{N} / \mathrm{O}$ ratio as a function of $\mathrm{O} / \mathrm{H}$. This result can be interpreted as due to the fact that $\mathrm{N}$ from massive stars is mainly a primary element, as originally suggested by Matteucci (1986). We explored this alternative and the resulting N/O evolution as a function of time, shown in Fig. 5, is rather flat and consistent with the nitrogen abundance observed in low-metallicity BCD galaxies. In this case, N/O cannot be used as a clock for the burst age.

The evolution of the $\mathrm{C} / \mathrm{O}$ ratio at the onset of the second burst is different from the behaviour of the N/O ratio. In particular, all the models shown in Fig. 5 seem to fall to a constant $\mathrm{C} / \mathrm{O}$ value (around -0.9 ) at the beginning of the second burst, then rising with different slopes, at variance with what happens with the $\mathrm{N} / \mathrm{O}$ evolution. The reason for this is that carbon is produced also in massive stars, thus the $\mathrm{C} / \mathrm{O}$ ratio after the onset of the second burst is mostly due to the massive stars produced by the second stellar generation, whereas the level of $\mathrm{N}$ is only determined by the IMS produced during the first burst.

In Fig. 7 the evolution of carbon, nitrogen and oxygen for model M500, case R, is shown. For this model we obtain good agreement with the observed abundances for an 


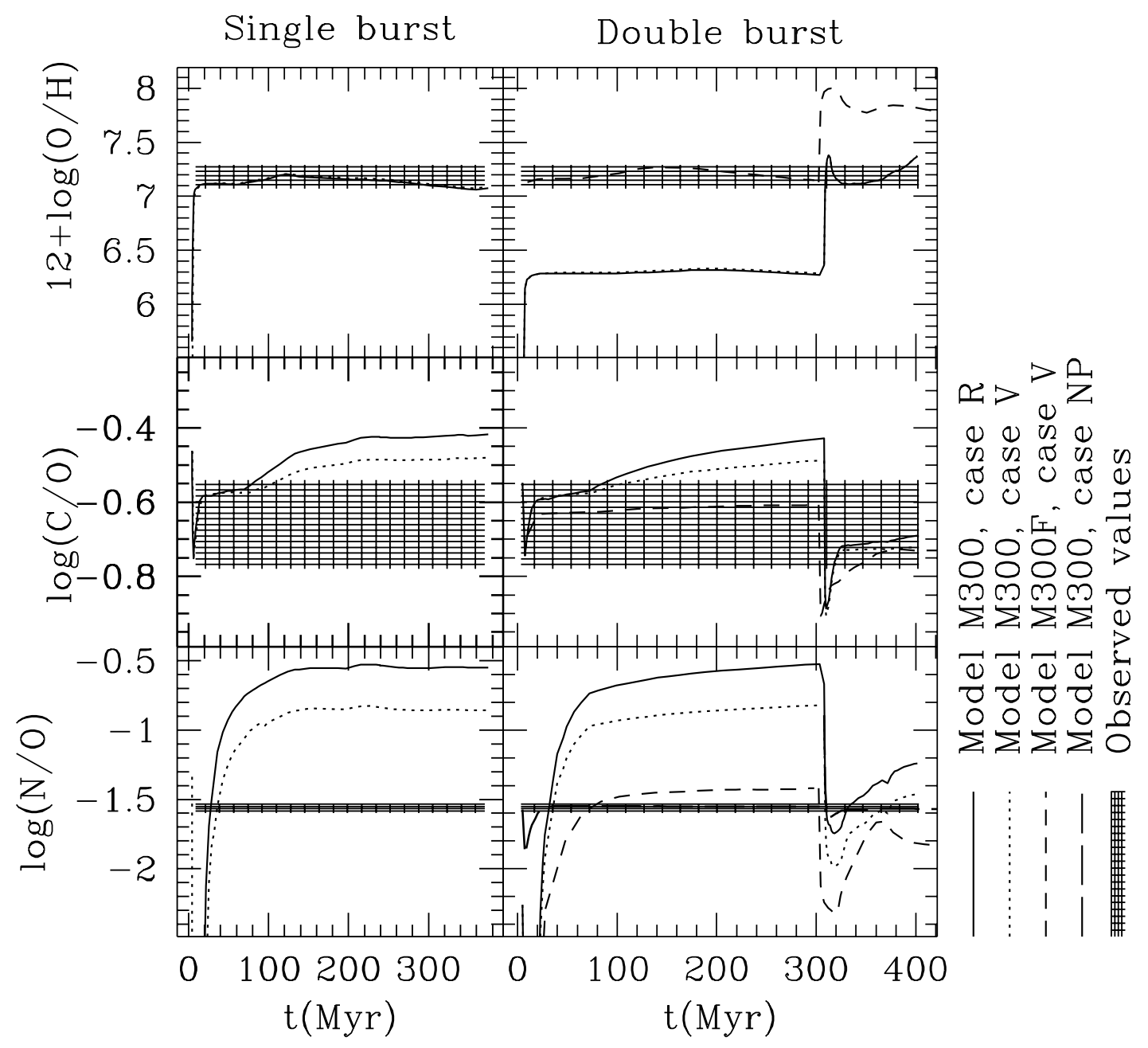

Fig. 5. Evolution of $\mathrm{O}, \mathrm{C}, \mathrm{N}$ for the single-burst model (left panels) and models M300 and M300F (right panels). In the bottom-right panel we show the evolution of N/O for a model (model NP) in which we assumed an "ad hoc" production of primary $\mathrm{N}$ in massive stars. The corresponding line is barely visible, because is completely embedded in the observational strip. The superimposed dashed areas represent the observative values found in literature for IZw18 (see text for references). Left panels represent the evolution of the single-burst model, with yields by RV81 (solid line) and VG97 (dotted line). In the right panel the solid line represent the evolution of model M300 with RV81 yields (case R), the dotted line the VG97 yields (case V), while the dashed line is the evolution of model M300F, with nucleosynthesis prescriptions by VG97 (case V).

age of the second burst of $\sim 6 \mathrm{Myr}$ or between $\sim 40 \mathrm{Myr}$ and $\sim 120 \mathrm{Myr}$, a far too large value. The results of this model slightly differ from those of model M300; in particular the evolution of nitrogen appears to be flatter than in model M300. In fact, at the onset of the second burst, the first generation of stars still produces and ejects metals into the ISM and the production of nitrogen due to the first starburst is larger for model M300. Moreover, the differences in the initial distribution of the ISM and in the luminosity of the starburst in models M300 and M500 have different dynamical consequences, which can explain the different N/O behaviours. In particular, after $\sim 150 \mathrm{Myr}$ the ejection efficiency of $\mathrm{N}$ is $\sim 0.91$ for the M500 model and $\sim 0.85$ for the M300, thus most of $\mathrm{N}$ produced in model M500 is lost and the evolution of N/O flattens.

In Fig. 8 the $[\alpha / \mathrm{Fe}]$ ratios for model M300, case $\mathrm{R}$, is shown compared to the results of the single-burst model. The $[\mathrm{O} / \mathrm{Fe}]$ ratios in the two-burst case are always lower outside than inside the galaxy, but the effect is less evident than in the one-burst case. Note that, after the first burst in model M300, no outflows are expected, thus no metals are present in the external region. The evolution of the $[\mathrm{O} / \mathrm{Fe}]$ ratio in the model M500 is shown in Fig. 9.

As evident from these figures, the overabundance of $\alpha$-elements lasts only for the first 20-30 Myr after the second burst and the maximum overabundance is of the order of 0.4 dex, which is in good agreement with some observations (Izotov et al. 1997 give $[\mathrm{O} / \mathrm{Fe}]=0.39 \pm 0.09$ ). Recently, Levshakov et al. (2001) with their mesoturbulent approach, found higher overabundances of $\alpha$ elements (they found $[\mathrm{Si}, \mathrm{Ar} / \mathrm{Fe}]=0.7 \pm 0.1$ ). Since there are some indications that the WW yields of iron are overestimated by a factor of 2 (Timmes et al. 1995), we tried to calculate $\alpha / \mathrm{Fe}$ ratios with reduced iron yields from massive stars and the results for $[\mathrm{Mg} / \mathrm{Fe}]$ and $[\mathrm{Si} / \mathrm{Fe}]$ evolution are shown in Fig. 10. As shown in this figure, 


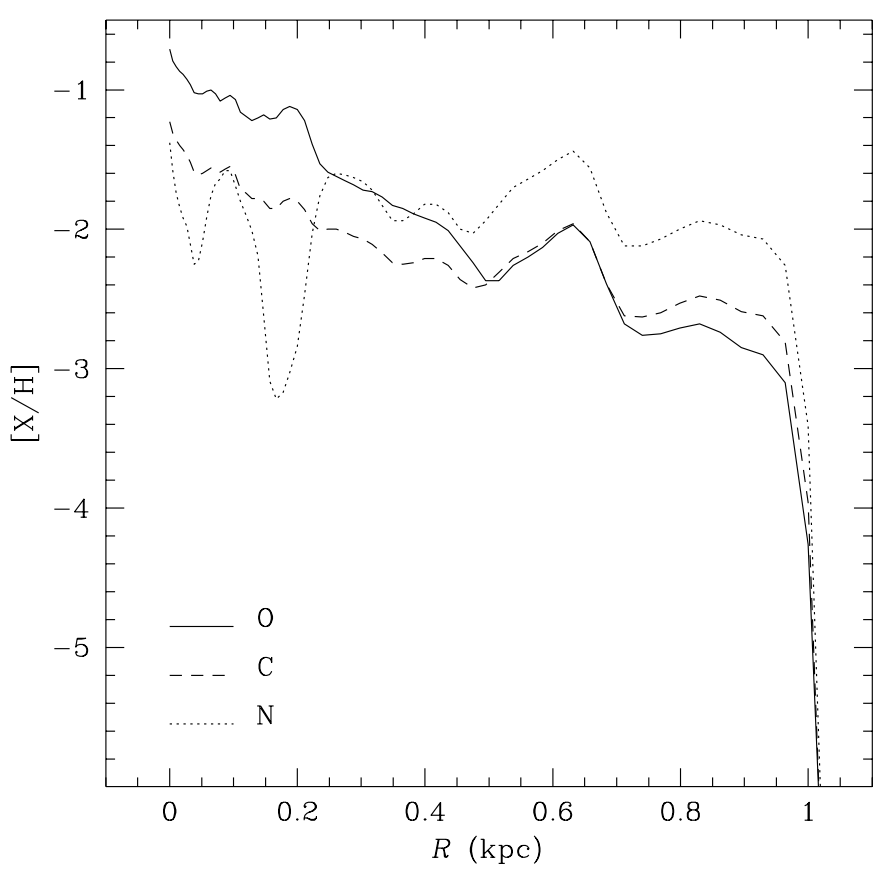

Fig. 6. Abundance ratios of oxygen (solid line), carbon (dashed line) and nitrogen (dotted line) relative to hydrogen, calculated using the column densities of the neutral medium for the model M300 after $\sim 6$ Myr. These values are calculated integrating along all the lines of sight, covering the whole computational grid, assuming the galaxy to be edge-on.

the overabundances of $\mathrm{Si}$ and $\mathrm{Mg}$ are larger than 0.6 dex after the second burst, and they last until $\sim 30 \mathrm{Myr}$ after the onset of the second burst.

As mentioned in the introduction, IZw18 provides useful information about the primordial helium abundance, because BCDs are the least chemically evolved galaxies known, so they are supposed to contain very little helium processed by stars after the big bang. Indeed, in our models, the variations of the helium mass fraction are at maximum of the order of $1 \%$, thus confirming the robustness of this approach in determining the primordial ${ }^{4} \mathrm{He}$ abundance.

\section{Discussion and conclusions}

In this paper we have studied the chemo-dynamical evolution of the gas as a consequence of two instantaneous starbursts, separated by a quiescent period, in a galaxy similar to IZw18. We have taken into account, in detail, both SN (Ia and II) feed-back and stellar yields. The main conclusions can be summarized as follows:

- In most of the explored cases a wind develops only during the second burst and the newly formed metals are ejected more efficiently than the pristine gas (mostly H), confirming previous results (Paper I; D'Ercole \& Brighenti 1999; MacLow \& Ferrara 1999).

- In particular, the metals produced by type Ia SNe are lost more efficiently than those produced by type II $\mathrm{SNe}$, due to the higher efficiency in energy transfer

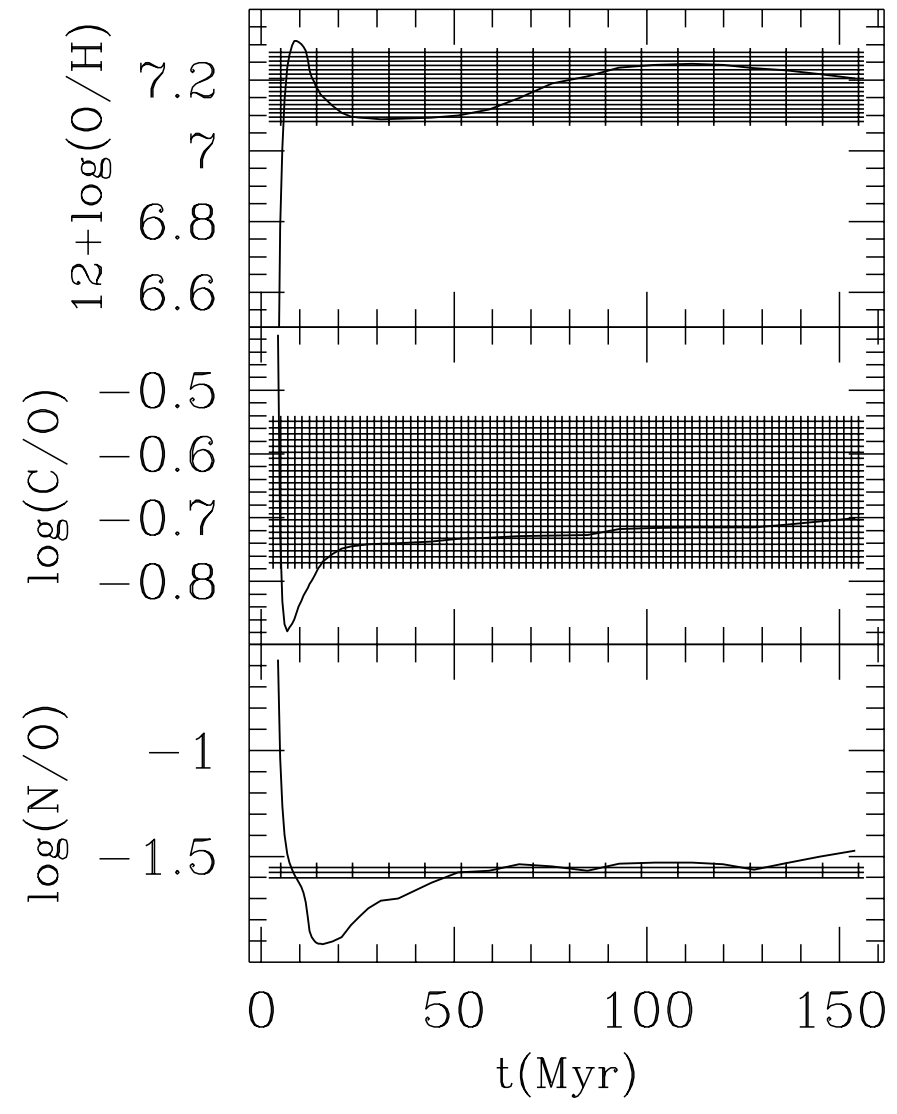

Fig. 7. Evolution of $\mathrm{O}, \mathrm{C}, \mathrm{N}$ for model M500. The time is calculated from the onset of the second burst. As in Fig. 5, the superimposed dashed areas represent the observative values found in literature for IZw18.

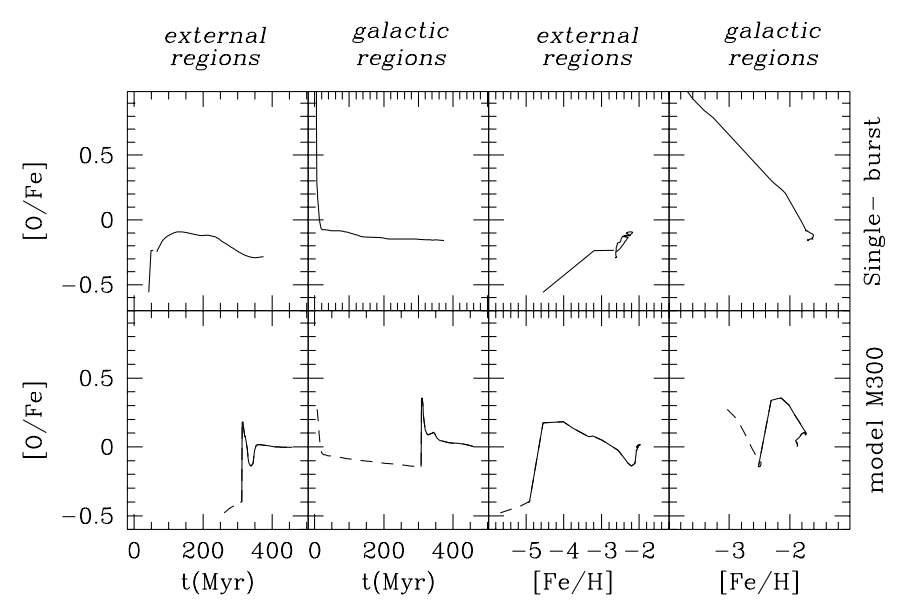

Fig. 8. O/Fe vs. time and vs. Fe/H for the single- burst model (upper panels) and for model M300, case R (lower panels). Dashed lines represent the chemical evolution of the first burst for model M300.

by type Ia SNe, which explode in an already hot and rarefied medium. However this effect, already found in Paper I where only one burst was assumed, is milder in the case of 2 bursts, since SNeII in the second burst are also likely to transfer more energy into the ISM.

- Our best model suggests that a first weak burst of star formation occurred roughly $300 \mathrm{Myr}$ ago, followed by 

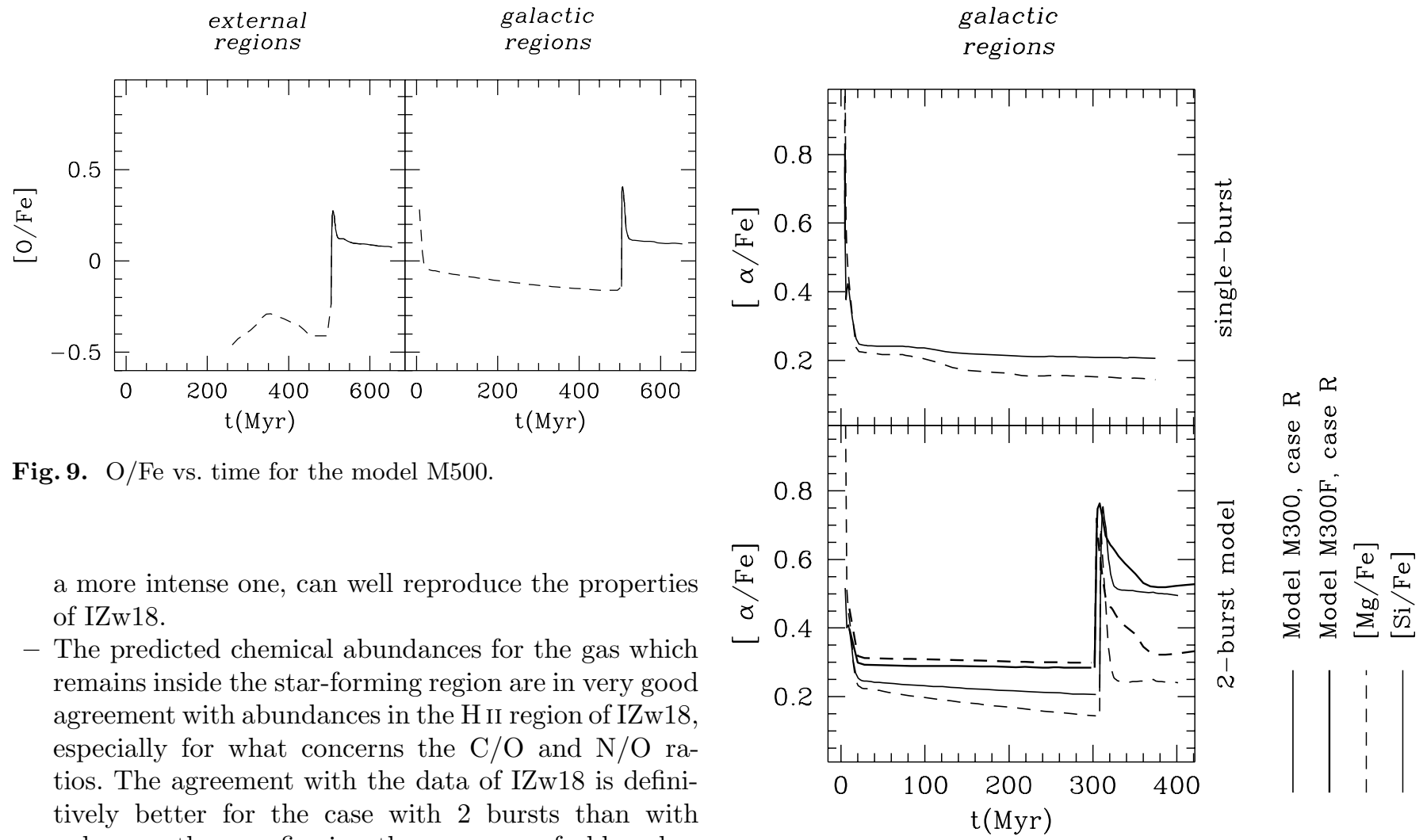

Fig. 9. O/Fe vs. time for the model M500.

a more intense one, can well reproduce the properties of IZw18.

- The predicted chemical abundances for the gas which remains inside the star-forming region are in very good agreement with abundances in the $\mathrm{H}$ II region of $\mathrm{IZw} 18$, especially for what concerns the $\mathrm{C} / \mathrm{O}$ and $\mathrm{N} / \mathrm{O}$ ratios. The agreement with the data of IZw18 is definitively better for the case with 2 bursts than with only one, thus confirming the presence of old underlying stellar populations (ATG, Östlin 2000). These results are in good agreement with observations either when using the chemical yields from low and intermediate mass stars by Renzini \& Voli (1981) or those by van den Hoek \& Groenewegen (1997).

- In the framework of the standard nucleosynthesis in massive stars we find two solutions for the age of the second burst: 1) an age of some tens of Myr (depending on the nucleosynthetic prescriptions adopted) and 2) an age of 4-6 Myr. This second solution is in good agreement with the spectral energy distribution models studied by MHK. If we assume that massive stars produce only primary $\mathrm{N}$, the situation changes since the right $\mathrm{N} / \mathrm{O}$ ratio is achieved already after 3-4 Myr after the first burst. Therefore, in this case we cannot use the N/O as a cosmic clock. However, the assumption of massive stars producing only primary $\mathrm{N}$ is perhaps too extreme. Therefore, we conclude that on the basis of chemo-dynamical results alone we cannot safely suggest the age of the present burst. As a consequence of this, we checked our burst age by computing the spectral energy distribution expected for the second burst, in the best model case. To do this we have computed the $U-B$ and $B-V$ colors by adopting the package Starburst99 (Leitherer et al. 1999), a web based software and data package designed to model spectrophotometric and related properties of star-forming galaxies. The results obtained with Starburst99 were then compared with the observed values of IZw18 (namely $U-B=-0.88 \pm 0.06$ and $B-V=-0.03 \pm 0.04$; van Zee et al. 1998) and they are shown in Fig. 11. From this figure one can see

Fig. 10. $[\mathrm{Mg} / \mathrm{Fe}]$ and $[\mathrm{Si} / \mathrm{Fe}]$ vs. time for the single-burst model (upper panel) and for model M300, case R and M300F, case $\mathrm{R}$ (lower panel), calculated with halved Fe yields from massive stars. Dashed lines represent the chemical evolution of the magnesium, while solid lines are the evolution of silicon.

that the outputs of Starburst99 are consistent with the observed $U-B$ only if the second burst has an age between 5.3 and $11 \mathrm{Myr}$, whereas the $B-V$ color is well reproduced if the age is between 23 and 44 Myr. However, the $B-V$ color is affected by the presence of the older stellar population (impossible to simulate with the Starburst99 package), whereas the $U-B$ color is dominated by young stars. Thus, these results seem to indicate that a very young age for the second burst (of the order of 5-6 Myr) is the most likely solution, at least in the hypothesis of an instantaneous burst of star formation. Moreover, we can also estimate the age of the second burst from dynamical considerations. In particular, in our best model the bubble has travelled a distance of $720 \mathrm{pc}$ in only 6-7 Myr from the beginning of the starburst. This distance is exactly that between the shell and the center of the NW H II region observed in IZw18 (Martin 1996). Martin (1996) herself suggests an age larger than the one derived here (see Table 4). This difference is due to the fact that Martin (1996) assumed an ISM distribution unmodified by the previous burst. In fact, the bubble expansion timescale strongly depends on the gas distribution. In conclusion, our results combined with the information we have from other studies, as summarized in Table 4, and taking into account the fact that real starbursts 
Table 4. Ages of the second burst from various sources.

\begin{tabular}{ccc}
\hline age $(\mathrm{Myr})$ & source & reference \\
\hline $5-6$ & model M300 & this paper \\
$40-70$ & model M300 & this paper \\
$\sim 4$ & model M300F & this paper \\
$\sim 6$ & model M500 & this paper \\
$40-120$ & model M500 & this paper \\
$6-7$ & bubble dynamics (M300) & this paper \\
\hline $5-11$ & $U-B$ & S99 \\
$23-44$ & $B-V$ & S99 \\
13.6 & $M_{B}$ & S99 \\
\hline $15-20$ & optical CMD & ATG \\
$15-20$ & NIR CMD & Ostlin $(2000)$ \\
$15-27$ & bubble dynamics & Martin $(1996)$ \\
$3-13$ & integrated spectra & MHK \\
\hline
\end{tabular}

Notes: we quote as S99 our own application of the Starburst99 (Leitherer et al. 1999) models to our examined cases. See text for details.

are not instantaneous, suggest that one can reasonably conclude that the age of the present burst should be in the range $5-15$ Myr.

- Models with a flat $(x=0.5)$ IMF produce much more oxygen after the first burst of star formation compared to models with the Salpeter (1955) IMF. For these models the only possible solution is a second burst of a very short age (around $4 \mathrm{Myr}$ ).

- The $[\alpha / \mathrm{Fe}]$ ratios in the two-burst case are always lower in the gas lost through the wind than in the gas which remains bound to the galaxy, thus creating an asymmetry. However this effect, already present in the one-burst case, is less strong here. The $[\alpha / \mathrm{Fe}]$ inside the galaxy is predicted to be higher than solar (overabundance of $\alpha$-elements) during the first $30 \mathrm{Myr}$ from the burst. In particular, we predict a value of $[\mathrm{O} / \mathrm{Fe}] \sim+0.4$ dex when the standard yields are adopted and a value of $[\mathrm{O} / \mathrm{Fe}] \sim+0.7-0.8 \mathrm{dex}$ when the $\mathrm{Fe}$ in massive stars is artificially lowered by a factor of two, since the Woosley \& Weaver (1995) yields for Fe seem to be overestimated. The last value of $[\mathrm{O} / \mathrm{Fe}]$ is in very good agreement with the abundance analysis of IZw18 by Levshakov et al. (2001).

- Finally, we computed the abundances of metals which should pertain to the HI gas and estimated that they are roughly lower by a factor of 2 compared to the abundances in the star forming region (H II), a prediction which should be tested on new FUSE data.

Before concluding we want to address some comments about the limits of the present model. In particular, the above results refer to the case of two subsequent instantaneous bursts of SF, the most common scenario usually attributed to the evolution of BCD. Until recently BCDs were supposed to undergo short and intense bursts

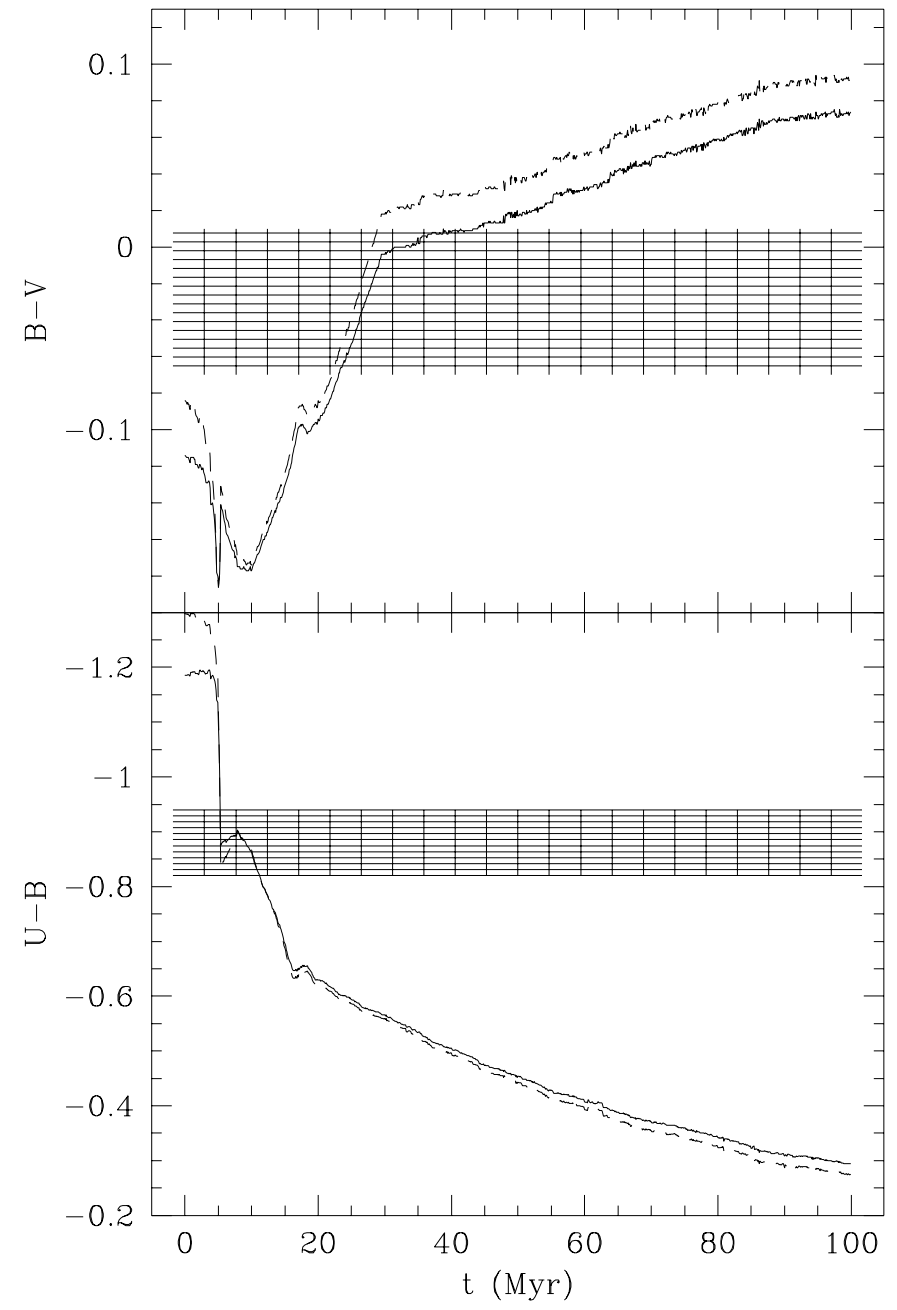

Fig. 11. $U-B$ and $B-V$ colors computed by means of Starburst99 (Leitherer et al. 1999), for the model M300 (solid line) and M300F (dashed line), compared with published observed values for IZw18, with relative error-bars (dashed areas).

of star formation, separated by long quiescent intervals, whereas dwarf irregulars and giant irregulars appeared to have a more continuous SF activity (e.g. Tosi 1999). For this reason, the vast majority of chemical evolution models for BCDs (e.g. Matteucci \& Tosi 1985; Pilyugin 1993; Bradamante et al. 1998) were computed assuming burst durations of $10^{8}$ years or less.

Nowadays, however, there is increasing evidence that also BCDs have rather a gasping SF, with long episodes of activity, separated by short quiescent intervals, if any. This continuity has been hypothesized by a few authors (e.g. Carigi et al. 1995; Legrand 2000), and has been supported by observations when the Hubble Space Telescope has allowed to resolve faint single stars even in galaxies outside the Local Group. The application of the method of synthetic color-magnitude diagrams (CMD) to derive the SF history in relatively distant BCD has provided fundamental information on the evolution of these systems. Deep CMD have been obtained from HST optical and infrared photometry of several BCD by different groups 
(e.g. Aloisi et al. 1999; Schulte-Ladbeck et al. 2001; Tosi et al. 2001) and have provided similar scenarios for the SF histories of the examined objects: the SF activity has started long ago (in all cases, at least as long ago as given by the maximum lookback time corresponding to the depth of the available photometry), but has been very intense only in a few galaxies. Standard episodes have a duration of several $10^{8}$ years and tend to overlap each other with no real quiescent phases in between, or fairly short ones (lasting only 5-10 Myr).

If the latter scenario turns out to apply in general to most BCD, the next fundamental step for a better understanding of their chemical evolution will be to take into account the effect on the ISM of SN explosions produced during long-lasting SF episodes. In fact, in most of the model solutions selected here, the predicted $\mathrm{N}$ abundances cross the observed range in a very short time interval (cf. Figs. 5 and 7). We expect that models with continuous SF will predict abundances remaining within the observed range for longer, more realistic timescales. Despite the much heavier computer requirements, we are currently working on this implementation in our treatment and the results will be presented in a forthcoming paper.

Acknowledgements. We like to thank the referee Dr. James Lequeux for his competent and useful suggestions that helped to significantly improve the clarity of this paper. This work has been partly supported by the Italian MIUR through Cofin2000.

\section{References}

Aloisi, A., Tosi, M., \& Greggio, L. 1999, AJ, 118, 302 (ATG)

Babul, A., \& Rees, M. J. 1992, MNRAS, 255, 346

Borriello, A., \& Salucci, P. 2001, MNRAS, 323, 285

Bradamante, F., Matteucci, F., \& D'Ercole, A. 1998, A\&A, 337,338

Carigi, L., Colin, P., Peimbert, M., \& Sarmiento, A. 1995, ApJ, 445, 98

Chang, R. X., Hou, J. L., Shu, C. G., \& Fu, C. Q. 1999, A\&A, 350,38

Davidson, K., \& Kinman, T. D. 1985, ApJS, 58, 321

Davidson, K., Kinman, T. D., \& Friedman, S. D. 1989, AJ, 97, 1591

D'Ercole, A., \& Brighenti, F. 1999, MNRAS, 309, 941

Dufour, R. J., \& Hester, J. J. 1990, ApJ, 350, 149

Dufour, R. J., Garnett, D. R., \& Shields, G. A. 1988, ApJ, 332, 752

Dufour, R. J., Esteban, C., \& Castañeda, H. O. 1996, ApJ, 471, L87

Garnett, D. R., Dufour, R. J., Peimbert, M., et al. 1995, ApJ, 449, L77

Garnett, D. R., Skillman, E. D., Dufour, R. J., \& Shields, G. A. 1997, ApJ, 481, 174

Heger, A., \& Langer, N. 2000, ApJ, 544, 1016

Hunter, D. A., \& Thronson, H. A. 1995, ApJ, 452, 238

Izotov, Y. I., \& Thuan, T. X. 1999, ApJ, 511, 639

Izotov, Y. I., Thuan, T. X., \& Lipovetsky, V. A. 1997, ApJS, 108,1

Izotov, Y. I., Chaffee, F. H., Foltz, C. B., et al. 1999, ApJ, 527, 757
Izotov, Y. I., Schaerer, D., \& Charbonnel, C. 2001a, ApJ, 549, 878

Izotov, Y. I., Chaffee, F. H., Foltz, C. B., et al. 2001b, Proc. of the XVII IAP Colloq. Gaseous Matter in Galaxies and Intergalactic Space [astro-ph/0109519]

Kniazev, A. Y., Pustilnik, S. A., Masegosa, J., et al. 2000, A\&A, 357, 101

Kunth, D., \& Östlin, G. 2000, A\&AR, 10, 1

Kunth, D., Lequeux, J., Sargent, W. L. W., \& Viallefond, F. 1994, A\&A, 282, 709

Kunth, D., Matteucci, F., \& Marconi, G. 1995, A\&A, 297, 634

Legrand, F. 2000, A\&A, 354, 504

Legrand, F., Kunth, D., Roy, J.-R., Mas-Hesse, J. M., \& Walsh, J. R. 2000, A\&A, 355, 891

Leitherer, C. 2001, to appear in the Proc. of Astrophysical Ages and Time Scales, ASP Conf. Ser., ed. T. von Hippel, N. Manset, \& C. Simpson

Leitherer, C., Schaerer, D., Goldader, J. D., et al. 1999, ApJS, 123,3

Lequeux, J., \& Viallefond, F. 1980, A\&A, 91, 269

Levshakov, S. A., Kegel, W. H., \& Agafonova, I. I. 2001, A\&A, 373,836

Lipovetsky, V. A., Chaffee, F. H., Izotov, Y. I., et al. 1999, ApJ, 519, 177

MacLow, M.-M., \& Ferrara, A. 1999, ApJ, 513, 142

Masegosa, J., Moles, M., \& Campos-Aguilar, A. 1994, 420, 576

Mas-Hesse, J. M., \& Kunth, D. 1999, A\&A, 349, 765 (MHK)

Matteucci, F. 1986, PASP, 98, 973

Matteucci, F. 1996, Fund. Cosm. Phys., 17, 283

Matteucci, F., \& Tosi, M. 1985, MNRAS, 217, 391

Mori, M., Ferrara, A., \& Madau, P. 2001 [astro-ph/0106107]

Nomoto, K., Thielemann, F.-K., \& Yokoi, K. 1984, ApJ, 286, 644

Olive, K. A., Skillman, E. D., \& Steigman, G. 1997, ApJ, 489, 1006

Östlin, G. 2000, ApJ, 535, L99

Östlin, G., Amram, P., Bergvall, N., et al. 2001, A\&A, 374, 800

Petrosian, A. R., Boulesteix, J., Comte, G., Kunth, D., \& LeCoarer, E. 1997, A\&A, 318, 390

Pettini, M., \& Lipman, K. 1995, A\&A, 297, L63

Pilyugin, L. S. 1993, A\&A, 277, 42

Recchi, S., Matteucci, F., \& D'Ercole, A. MNRAS, 322, 800, Paper I

Reimers, D. 1975, Mem. R. Sci. Liege Ser. 6, 8, 369

Renzini, A., \& Voli, M. 1981, A\&A, 94, 175 (RV81)

Romano, D., Matteucci, F., Salucci, P., \& Chiappini, C. 2000 , ApJ, 539, 235

Saitō, M., Sasaki, M., Ohta, K., \& Yamada, T. 1992, PASJ, 44, 593

Salpeter, E. E. 1955, ApJ, 121, 161

Searle, L., \& Sargent, W. L. W. 1972, ApJ, 173, 25

Schulte-Ladbeck, R. E., Hopp, U., Greggio, L., Crone, M. M., \& Drozdovsky, I. O. 2001, AJ, in press

Skillman, E. D., \& Kennicutt, R. C. J. 1993, ApJ, 411, 655

Skillman, E. D., Terlevich, R., Teuben, P. J., \& van Woerden, H. 1988, A\&A, 198, 33

Skillman, E. D., Terlevich, E., \& Terlevich R. 1998, in Primordial Nuclei and their Galactic Evolution, ed. N. Prantzos, M. Tosi, \& R. van Steiger (Dordrecht: Kluwer), 105 
Terlevich, E., Skillman, E. D., \& Terlevich, R. 1996, in The Interplay between Massive Star Formation, the ISM and Galaxy Evolution, ed. D. Kunth, B. Guiderdoni, M. Heydari-Malayeri, \& T. Thuan (Gif-sur-Yvette: Éd. Frontières), 395

Timmes, F. X., Woosley, S. E., \& Weaver, T. A. 1995, ApJS, 98,617

Tosi, M. 1999, in Dwarf galaxies and cosmology, ed. T. X. Thuan, C. Balkowski, V. Cayatte, \& J. Tran Thanh Van (Éditions Frontières, Gif-sur-Yvette, France), 443

Tosi, M. 2001, in Dwarf Galaxies and their Environment, ed. K. S. de Boer, R. J. Dettmar, \& U. Klein (Shaker Verlag, De), in press

Tosi, M., Greggio, L., Marconi, G., \& Focardi, P. 1991, AJ, 102, 951 van den Hoek, L. B., \& Groenewegen, M. A. T. 1997, A\&AS, 123, 305 (VG97)

van Zee, L. 2001, AJ, 121, 2003

van Zee, L., Westpfahl, D., \& Haynes, M. P. 1998, AJ, 115, 1000

Viallefond, F., Lequeux, J., \& Comte G. 1987, in Starbursts and Galaxy Evolution, Proc. of the Twentysecond Moriond Astrophysics Meeting (Gif-sur-Yvette: Éd. Frontières), 139

Vidal-Madjar, A., Kunth, D., Lecavelier des Etangs, A., et al. 2000, ApJ, 538, L77

Vilchez, J. M., \& Iglesias-Páramo, J. 1998, ApJ, 508, 248

Woosley, S. E., \& Weaver, T. A. 1995, ApJS, 101, 181 (WW) 